\title{
Low Incidence of Clinically Significant Heparin Induced Thrombocytopenia after Cardiopulmonary Bypass Surgery
}

Abhinav B Chandra*, Navneet Mittal, Shilpa Sambidi, Anuradha Belur, Swati Pathak, Himanshu Pathak and Yiqing Xu

Division of Hematology and Oncology, Department of Internal Medicine, Maimonides Medical Center, Brooklyn, New York, USA

\begin{abstract}
Introduction: Thrombocytopenia after cardiopulmonary bypass (CPB) is common, and the diagnosis of heparin induced thrombocytopenia (HIT) remains a challenge in clinical practice despite the guidance from the " $4 \mathrm{~T}$ " diagnostic scoring criteria and the laboratory tests.

Objectives: This study aims to evaluate (i) the temporal laboratory characteristics of thrombocytopenia after $\mathrm{CPB}$ and (ii) the incidence of clinically significant HIT based on clinical assessment.

Methods: A retrospective data review of patients undergoing coronary artery bypass graft surgery or valve surgery who were also placed on bypass pump and received intra-operative heparin.

Results: Among 450 study patients, $142(31.5 \%)$ patients developed decrease of platelet counts at least $33 \%$ below base line following surgery, with a median degree of $61 \%$. The initial platelet nadir occurred between day 0 to day 4 (median 1 day). The cumulative percentage of patients demonstrating recovery of their platelet count was $44 \%$ by day $4,80 \%$ by day 5 and $100 \%$ by day 10 . Only 9 patients $(2 \%)$ showed a second decrease in platelet count. By clinical assessment, including clinical outcome, platelet recovery, Doppler test results and the need for use of direct thrombin inhibitors, not a single case of clinically significant HIT was diagnosed. Heparin associated platelet factor 4 (H-PF4) antibody test was positive in $10 \%$ and $0 \%$ of patients who developed platelet count decrease in the first and second phase, respectively.
\end{abstract}

Conclusion: A very low incidence of clinically significant HIT after CABG using clinical assessment was observed and further prospective trials are warranted to confirm this observation.

Keywords: Thrombocytopenia; Cardiopulmonary bypass surgery; Heparin-induced thrombocytopenia; Deep venous thrombosis

Abbreviation: CPB: Cardiopulmonary Bypass; HIT: HeparinInduced Thrombocytopenia; H-PF4: Heparin Associated Platelet Factor 4; DVT: Deep Venous Thrombosis; ELISA: Enzyme-Linked Immuno Assays; SRA: Serotonin Release Assay; CPT: Current Procedural Terminology

\section{Introduction}

Thrombocytopenia after Cardio-Pulmonary bypass (CPB) is a common occurrence due to multiple causes. Surgery itself is a major reason which leads to mechanical destruction of platelets and hemodilution in the bypass circuit. The other important cause is Heparin-induced thrombocytopenia (HIT). HIT has been reported to occur in $2-3 \%$ patients after CPB [1,2], is associated with significant risk of thrombotic complications up to 38 to $81 \%[1,3]$, and a mortality rate of $28 \%$ if left untreated [4].

In clinical practice, it is important to accurately diagnose HIT and to promptly start anticoagulation therapy using direct antithrombin inhibitors [1,2,5]. Clinical criteria predictive of HIT diagnosis in general patient population as well as in the post CPB setting have been developed [6,7]; patients who have a second fall in platelet counts between 5 to 14 days after initial heparin exposure [6,7], or patient who have a persistent thrombocytopenia at and after 5 days of CPB [7] are considered to have high incidence of HIT. The screening laboratory tests used for detecting the presence of anti-heparin related platelet factor 4 (H-PF4) antibodies by Enzyme-linked immunoassays (ELISA) assay carries a high false positive rate [8,9], and the Serotonin Release assay (SRA), a gold standard for HIT diagnosis, has a long turn around time, usually not available for immediate guidance of therapy [10].

During our hematology consultation service in a community hospital with high volume cardiac surgery, we noticed a very low rate of HIT in post CABG patients. We carried out this retrospective study to evaluate the incidence of clinically significant HIT, focusing on using clinical assessment in supplementation of laboratory tests.

\section{Patients and Methods}

\section{Patient selection}

Using Current Procedural Terminology (CPT) codes, the Maimonides Medical Center database of all cardiac surgical cases in 2008 was searched for patients undergoing coronary artery bypass graft surgery and valve surgery, and those patients who were placed on cardiopulmonary bypass machine during the surgery and received intra-operative heparin were eligible. During 2008, more than 800 patients were eligible and the first 450 patients on the computer generated list were selected as the study population. Demographics and medical records on the time course of thrombocytopenia, laboratory tests for HIT, Doppler tests for DVT, pharmacy record for the use of

${ }^{*}$ Corresponding author: Abhinav B Chandra, Division of Hematology and Oncology, Department of Internal Medicine, Maimonides Medical Center, Brooklyn, 4802 Tenth Avenue, Brooklyn, NY 11219, New York, USA, Tel: 718765-2600; Fax: 718-765-2630; E-mail: abhinavbck@hotmail.com

Received October 23, 2013; Accepted December 10, 2013; Published Decembe 12, 2013

Citation: Chandra AB, Mittal N, Sambidi S, Belur A, Pathak S, et al. (2013) Low Incidence of Clinically Significant Heparin Induced Thrombocytopenia after Cardiopulmonary Bypass Surgery. J Blood Disorders Transf 5: 180. doi: 10.4172/2155-9864.1000180

Copyright: (C) 2013 Chandra AB, et al. This is an open-access article distributed under the terms of the Creative Commons Attribution License, which permits unrestricted use, distribution, and reproduction in any medium, provided the original author and source are credited. 
Citation: Chandra AB, Mittal N, Sambidi S, Belur A, Pathak S, et al. (2013) Low Incidence of Clinically Significant Heparin Induced Thrombocytopenia after Cardiopulmonary Bypass Surgery. J Blood Disorders Transf 5: 180. doi: 10.4172/2155-9864.1000180

Page 2 of 4

direct thrombin inhibitors, and ultimate outcomes of discharge or death were reviewed. The platelet count decrease and recovery trend on individual patients were recorded.

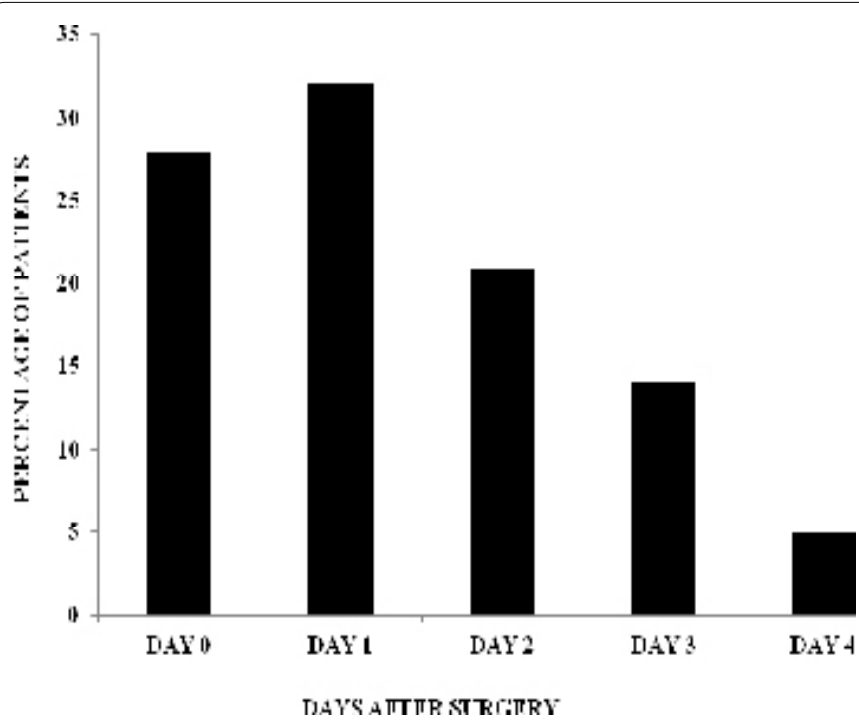

Figure 1: Bar graph showing percentage of patients reaching platelet nadir on days following surgery. X-axis- Days after surgery; Y-axis- Percentage of patients having platelet nadir.
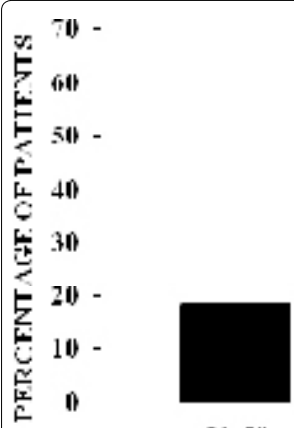

$31-50$

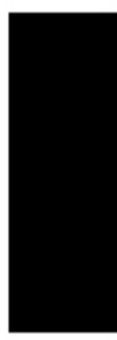

$51-70$

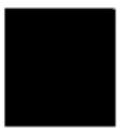

$71-100$

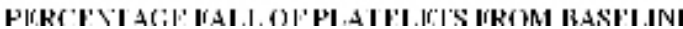

Figure 2: Bar graph showing percentage of patients having percentage fall of platelets from baseline. X-axis- Percentage fall of platelets from baseline Y-axis- Percentage of patients.

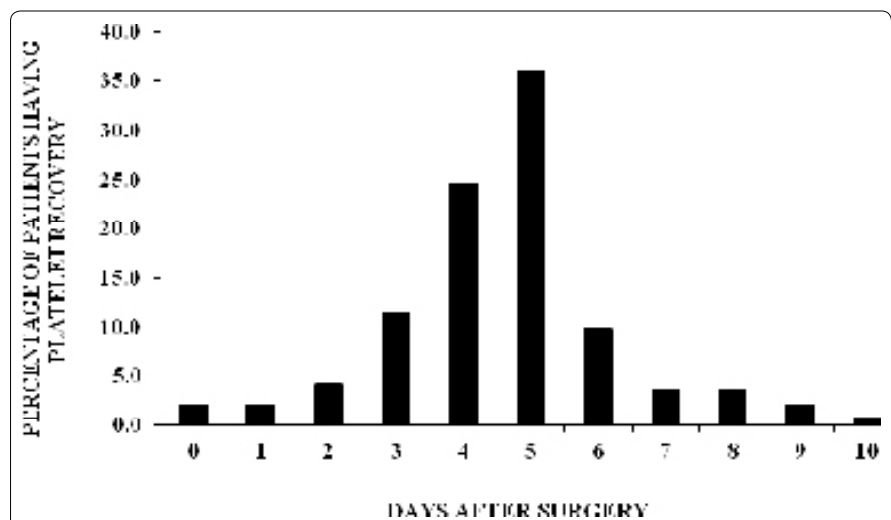

Figure 3: Graph showing percentage of patients having platelet recovery days following surgery. X-axis- Days after surgery; Y-axis- Percentage of patients having platelet recovery.

\section{Thrombocytopenia definitions}

Thrombocytopenia was defined as an initial platelet decrease greater than $33 \%$ from the patient's baseline and an absolute value below 100,000 occurring up to 10 days of surgery. The time from surgery to platelet nadir was recorded as time to nadir (in days). The time to initial recovery was defined as days post surgery when platelet count reached $50 \%$ above the nadir, and the time to final recovery was defined as days post surgery and one of the following: (i) platelet counts reached $75 \%$ of the patient's baseline, (ii) platelet count above 150,000, or (iii) upward trend in those who were discharged before full recovery. An episode of second platelet decrease was defined as any degree of platelet fall after initial recovery.

\section{Results}

\section{Characteristics of thrombocytopenia}

One hundred forty two patients (31.5\%) developed thrombocytopenia after surgery defined as first platelet nadir; among them, $40(28 \%)$ patients exhibited platelet nadir on the day of surgery (day 0 ) and showed immediate post-operation recovery. Other patients demonstrated further decrease in platelet counts after the day of operation, with $45(32 \%)$ patients reaching platelet nadir on day 1, 30 (21\%) patients on day 2, 20 (14\%) patients on day 3 and 7 (5\%) patients on day 4 (Figure 1). The median time to nadir was 1 day. The degree of platelet decrease ranged from $34 \%$ to $97 \%$, and the median was $61 \%$ (Figure 2). The initial platelet recovery started on day 0 and it took 10 days for all patients to achieve complete platelet recovery. In particular, $44 \%$ of patients showed recovery of their platelets by day $4,80 \%$ by day 5 with $36 \%$ recovering on day 5 itself, and $20 \%$ recovering after day 5 (Figure 3). Patient characteristics are summarized in Table 1.

Nine patients showed a second decrease in platelet count after initial recovery from the first fall, the nadir among these patients occurred from post-op days 2 to 25 and recovery started after 1 day. The recovery was complete in all patients by day 29 except in one patient, who subsequently died due to sepsis and his platelet count never recovered.

\section{Testing of heparin-induced thrombocytopenia}

H-PF4 antibody ELISA test was sent on 40 patients who demonstrated first platelet nadir after surgery. The selection for testing appeared to be random, and in 39 patients, the H-PF4 test was sent before or on day 5. Four patients (10\%) revealed positive H-PF4 antibody ELISA assay while the Serotonin release assay (SRA) test was only performed on one of the four patients and it was negative. H-PF4 antibody ELISA test was sent on five of the nine patients who demonstrated second platelet nadir, and all were negative ( $0 \%)$.

\section{Clinical assessment}

Doppler studies performed during the hospitalization of the above study population were reviewed and the results are summarized in Table 2. Those tests were triggered by abnormal physical exams. Among the patients who showed first platelet decrease after surgery, none of the patients who tested positive for H-PF4 antibody ELISA assay were found to have DVT; and none of the patients who were not tested for H-PF4 antibody had documented DVT. In contrast, 4 cases of DVT were found in the 36 patients who were tested negative for $\mathrm{H}-\mathrm{PF} 4$ antibody ELISA assay. Among the 9 patients who showed a second decrease in platelet count after initial recovery from the first fall, 2 of patients who tested positive for H-PF4 antibody ELISA assay were examined by Doppler studies and neither was positive. 


\begin{tabular}{|c|c|}
\hline Characteristics & Number of Patients \\
\hline Eligible patients & 450 \\
\hline Patients developing thrombocytopenia & 142 \\
\hline Male & 93 \\
\hline Female & 49 \\
\hline Intraoperative Heparin use & 450 \\
\hline Patients receiving platelet transfusion & 47 \\
\hline Median Age & 71 years (Range 41-90 years) \\
\hline
\end{tabular}

Table 1: Patient characteristics.

\begin{tabular}{|c|c|c|}
\hline & $\begin{array}{c}\text { H-PF4 antibody } \\
\text { tests }\end{array}$ & $\begin{array}{c}\text { DVT confirmed by } \\
\text { Doppler }\end{array}$ \\
\hline $\begin{array}{c}\text { Thrombocytopenia } \\
\text { after surgery }\end{array}$ & $\begin{array}{c}\text { Positive } n=4 \\
\text { Negative } n=36\end{array}$ & 0 \\
$\left(1^{\text {st }}\right.$ nadir $)$ & Not done $\mathrm{n}=102$ & 4 \\
\hline Thrombocytopenia & Positive $\mathrm{n}=0$ & 0 \\
after initial recovery & Negative $\mathrm{n}=2$ & 2 \\
$\left(2^{\text {nd }}\right.$ nadir $)$ & Not done $\mathrm{n}=7$ & 5 \\
\hline
\end{tabular}

Table 2: Patients Subgroups with Testing for DVT.

No use of direct thrombin inhibitors as anticoagulation therapy was documented in any of those patients either from the first or second platelet nadir.

One hundred forty one patients demonstrated platelet recovery. Six patients in the second nadir group (total 9 patients) were found to have concurrent sepsis, and all but one showed platelet recovery without anticoagulation therapy. Only one patient died of sepsis.

\section{Discussion}

In this retrospective study, we examined the incidence of HIT and HIT associated thrombosis in patients who developed thrombocytopenia after on pump CABG using clinical assessments including (i) the correlation of DVT with laboratory tests, (ii) the use of anticoagulation with direct thrombin inhibitors and (iii) the clinical outcome of platelet recovery. We did not find a single definitive case of HIT that could be diagnosed out of the 450 randomly selected patients. Our result was derived from data in a single community hospital with a high volume cardiac bypass surgery service, but no ready assess to SRA tests.

The above clinical assessment parameters are pertinent for the clinical diagnosis of HIT. New thromboembolism appears as a clinical feature in approximately half of all individuals diagnosed of HIT [1,3]. If not treated with alternative parenteral anticoagulation [11], even after heparin cessation, asymptomatic patients with isolated HIT will have $50 \%$ risk of developing HIT in the next 30 days, and will have $28 \%$ of mortality with persistent thrombocytopenia $[1,3,4]$. In this study, we did not have all the information of patient mortality; however the platelet recovery data served as a good surrogate.

DVT is not only a cardinal feature of HIT, but also a common diagnosis in the post operative setting unrelated to HIT. None of the patients tested positive for H-PF4 antibody, or not tested at all demonstrated DVT by Doppler exams. On the other hand, the only 4 cases of DVT were found in the group of patients who had negative HIT test results (Table 2). As a negative HIT PF4 antibody test carries a high negative predictive value for HIT [12], it is unlikely that those patients had undiagnosed HIT. Collectively, the above data argues against under-diagnosis of HIT based on clinical assessment in this study.

Our result of very low incidence of HIT after on pump CABG aligns with a recent publication showing a $0.2 \%$ HIT rate among over 10,000 patients undergoing cardiac surgery within a time period of 8 years [13], but is not consistent with previous published other reports documenting HIT incidence of 1-3\% [1,2]. One of the major differences between our study and the others is the lack of HIT test data on all patients, or all of the high risk patients meeting the scoring criteria [7]. This difference represents the real world situation in community practice where the selection of testing for HIT is sporadic. However determination predominantly based on HIT laboratory tests may lead to over-diagnosis. The H-PF4 ELISA test has a high false positive results [8,9], and it appears to be particular so in the patient population after CABG where as high as $50 \%$ positive rate was detected by post operation day $5[8,9]$. In addition, many patients going for CABG may already carry a baseline positive antibody titer [8]. On the other hand, SRA assay is widely considered a more specific test but is not readily available in the community. Even more surprisingly, in one prospective study where every unselected patient was tested for SRA, SRA could be positive in up to $12-13 \%$ patients after CPB, which has no correlation with platelet counts or the use of anticoagulation therapy [14]. In addition, another study also showed positive HIT PF4 antibody detectable by SRA in $5 \%$ and $13 \%$ of patients before and after cardiac surgery [9]. If SRA test result is solely used for diagnosis of HIT, the HIT rate post $\mathrm{CPB}$ also would be much higher, again representing over-diagnosis. Therefore, the diagnostic consideration of HIT should incorporate clinical outcomes data and not just based on laboratory tests.

Guidelines for selection of patients to undergo HIT testing have been developed, and the best studied guideline is the 4 " $\mathrm{T}$ " criteria [6,7]. In patients after CPB surgery, those who demonstrate persistent thrombocytopenia after post CPB day 4, or develop a second platelet decrease following initial recovery [7] appeared to harbor high prevalence of HIT. Although sensitive in detecting HIT, this system is limited by a positive predictive value of $9-17 \%$ and modest interobserver agreement $[5,6,12,15,16]$. Our results reinforced this notion and provided explanations for such a low positive predictive value. First, we examined the temporal trend of platelet decrease and recovery in individual patients instead of taking average platelet counts of the group on a given day, and our data illustrated that only $44 \%$ of patients completed platelet recovery by day 4 ; more than $50 \%$ of the patients continued to show platelet recovery on day 5 and later until day 10. Those patients would all be eligible for HIT testing if no other explanations for thrombocytopenia exist under the " $4 \mathrm{~T}$ " criteria, but they are actually still recovering from the bypass machine mechanical damage during day 5 to 10 . Second, patients developing second phase of platelet decrease are considered high risk for HIT, but our results indicated that the main cause of thrombocytopenia in this case is sepsis.

In conclusion, our study demonstrated a very low incidence of clinically significant HIT using clinical assessment. We hope our study could provide some assurance for community hematologists and cardiothoracic surgeons who fear to miss a true HIT diagnosis; the result of our study really call for further prospective validation and improvement of diagnostic tools for HIT as a whole so that overdiagnosis and over-treatment of HIT in the post-CABG setting can be avoided.

\section{Acknowledgement}

We cordially thank $\mathrm{Dr}$. Alan Astrow and Dr. Yiwu Huang in Division of Hematology and Oncology, Department of Internal Medicine, Maimonides Medical Center for their critical reading and helpful comments for this manuscript.

\section{References}

1. Warkentin TE, Greinacher A (2003) Heparin-induced thrombocytopenia and cardiac surgery. Ann Thorac Surg 76: 2121-2131. 
Citation: Chandra AB, Mittal N, Sambidi S, Belur A, Pathak S, et al. (2013) Low Incidence of Clinically Significant Heparin Induced Thrombocytopenia after Cardiopulmonary Bypass Surgery. J Blood Disorders Transf 5: 180. doi: 10.4172/2155-9864.1000180

2. Pouplard C, Regina S, May MA, Gruel Y (2007) Heparin-induced thrombocytopenia: a frequent complication after cardiac surgery. Arch Mal Coeur Vaiss 100: 563-568.

3. Warkentin TE, Kelton JG (1996) A 14-year study of heparin-induced thrombocytopenia. Am J Med 101: 502-507.

4. Walls JT, Curtis JJ, Silver D, Boley TM, Schmaltz RA, et al. (1992) Heparininduced thrombocytopenia in open heart surgical patients: sequelae of late recognition. Ann Thorac Surg 53: 787-791.

5. Cuker A (2011) Heparin-induced thrombocytopenia: present and future. $J$ Thromb Thrombolysis 31: 353-366.

6. Lo GK, Juhl D, Warkentin TE, Sigouin CS, Eichler P, et al. (2006) Evaluation of pretest clinical score (4T's) for the diagnosis of heparin-induced thrombocytopenia in two clinical settings. J Thromb Haemost 4: 759-765.

7. Lillo-Le Louët A, Boutouyrie P, Alhenc-Gelas M, Le Beller C, Gautier I, et al. (2004) Diagnostic score for heparin-induced thrombocytopenia after cardiopulmonary bypass. J Thromb Haemost 2: 1882-1888.

8. Visentin GP, Malik M, Cyganiak KA, Aster RH (1996) Patients treated with unfractionated heparin during open heart surgery are at high risk to form antibodies reactive with heparin: platelet factor 4 complexes. J Lab Clin Med128: 376-383.

9. Bauer TL, Arepally G, Konkle BA, Mestichelli B, Shapiro SS, et al. (1997) Prevalence of heparin-associated antibodies without thrombosis in patients undergoing cardiopulmonary bypass surgery. Circulation 95: 1242-1246.
10. Sheridan D, Carter C, Kelton JG (1986) A diagnostic test for heparin-induced thrombocytopenia. Blood 67: 27-30.

11. Greinacher A, Janssens U, Berg G, Böck M, Kwasny H, et al. (1999) Lepirudin (recombinant hirudin) for parenteral anticoagulation in patients with heparininduced thrombocytopenia. Heparin-Associated Thrombocytopenia Study (HAT) investigators. Circulation 100: 587-593.

12. Bryant A, Low J, Austin S, Joseph JE (2008) Timely diagnosis and managemen of heparin-induced thrombocytopenia in a frequent request, low incidence single centre using clinical 4T's score and particle gel immunoassay. $\mathrm{Br} \mathrm{J}$ Haematol 143: 721-726.

13. Crowther MA, Cook DJ, Albert M, Williamson D, Meade M, et al. (2010) The 4Ts scoring system for heparin-induced thrombocytopenia in medical-surgical intensive care unit patients. J Crit Care 25: 287-293.

14. Konkle BA, Bauer TL, Arepally G, Cines DB, Poncz M, et al. (2001) Heparininduced thrombocytopenia: bovine versus porcine heparin in cardiopulmonary bypass surgery. Ann Thorac Surg 71: 1920-1924.

15. Pouplard C, Gueret P, Fouassier M, Ternisien C, Trossaert M, et al. (2007) Prospective evaluation of the '4Ts' score and particle gel immunoassay specific to heparin/PF4 for the diagnosis of heparin-induced thrombocytopenia. J Thromb Haemost 5: 1373-1379.

16. Denys B, Stove V, Philippé J, Devreese K (2008) A clinical-laboratory approach contributing to a rapid and reliable diagnosis of heparin-induced thrombocytopenia. Thromb Res 123: 137-145.
Citation: Chandra AB, Mittal N, Sambidi S, Belur A, Pathak S, et al. (2013) Low Incidence of Clinically Significant Heparin Induced Thrombocytopenia after Cardiopulmonary Bypass Surgery. J Blood Disorders Transf 5: 180. doi: $10.4172 / 2155-9864.1000180$ 